\title{
Far Future Colliders and Required R\&D Program
}

\author{
V. Shiltsev
}

FNAL, PO Box 500, Batavia, IL 60510 USA

\begin{abstract}
Particle colliders for high energy physics have been in the forefront of scientific discoveries for more than half a century. The accelerator technology of the collider has progressed immensely, while the beam energy, luminosity, facility size and the cost have grown by several orders of magnitude. The method of colliding beams has not fully exhausted its potential but its pace of progress has greatly slowed down. In this paper we very briefly review the R\&D toward near future colliders and make an attempt to look beyond the current horizon and outline the changes in the paradigm required for the next breakthroughs.
\end{abstract}

Keywords: colliders, energy frontier, crystals, muon beams

PACS: 41.75.Lx, 41.75.Jv, 52.38.Kd, 29.20.db, 29.20.Ej

\section{COLLIDERS BEYOND 2030: LIMITATIONS}

Our forecast on the future of colliders beyond the 2030's will require several assumptions: on the available resources, on the desired science reach, and on the possible ways to the goal. Let us start with the money. As of today, the world's particle physics research budget is some 3B $\$$. That is about $1.2 \%$ of the world's total spending on basic science research (about 250B \$). For comparison, global R\&D funding of some $1,400 \mathrm{~B} \$$ is about $2 \%$ of the world's GDP of $\sim 70,000 \mathrm{~B} \$$ (all numbers from [1]). We argue that the era of the exponential expansion for most sciences is gone, and in the particular case of the high energy particle physics, the era of much more modest growth or even slow down of the financial support had began in the 1990's - see Fig.1. So, we shall base our predictions on the assumption that the field will stay approximately within the current financial limits, while still vigorously exploring new methods and directions.

The number of colliding beam facilities under discussion at present [2], can be distributed in several logarithmically broad categories, depending on the cost of construction:
Category I (under $\sim 0.3 \mathrm{~B} \$$ )
NICA, ENC
Category II $(0.3 \mathrm{~B} \$-1 \mathrm{~B} \$)$
Super-B factories, Tau-Charm factory, eRHIC, ELIC
Category III (1B\$ - 3B\$)
Category IV (3B \$-10B\$)
Category V (10B\$ - 30B \$)
Higgs factory, HL-LHC
HE-LHC, LHeC, Muon Collider, Higgs factory-ILC
ILC, CLIC

From what we know now, the Category I and II colliders can be built by one country, with relatively modest international contribution; Category III - requires significant cooperation at least within one of the big regions (Europe, Asia, America); Category IV machines will arguably need close cooperation of two or all three regions; while the Category V colliders must be truly international. Following our basic assumption (that the particle physics budget will not grow much beyond $\sim 3 \mathrm{~B} \$$ in current prices), it is hard to imagine that any of the projects in Category $\mathrm{V}$ are possible, especially given that their energy reach being only comparable to the LHC. Category III machines appear totally feasible financially, but they only add to exploration of the phenomena discovered by the LHC, thus, there always will be an alternative "just run the LHC longer". Finally, we are left with Category IV colliders which appear to be on the border of financial affordability. There is a problem, though, with them - they have limited energy reach, equivalent to some $5 \mathrm{TeV}$ c.m. energy in parton collisions (that is the Muon Collider energy, and the HE-LHC c.m. energy divided by an equivalent number of partons in proton $33 /(6-10)=3-5 \mathrm{TeV})$. It is not whatsoever certain whether there be a demanding physics case for such kind of c.m. energies to justify the required significant investment. 
Below we explore possibilities for physics reach of far future colliders within approximate budget of 10B\$ (in current prices). The cost models of the modern colliders are quite complicated and beyond the scope of this report, but one may safely assume that the future facility of such cost shall not exceed $10 \mathrm{of} \mathrm{km}$ in length and simultaneously shall require less than 10 to few 10's of MW of beam power (or correspondingly stay under $\sim 100 \mathrm{MW}$ of AC wall power consumption). Can such colliders reach c.o.m. energies orders of magnitude beyond current - namely, 100-1000 TeV?

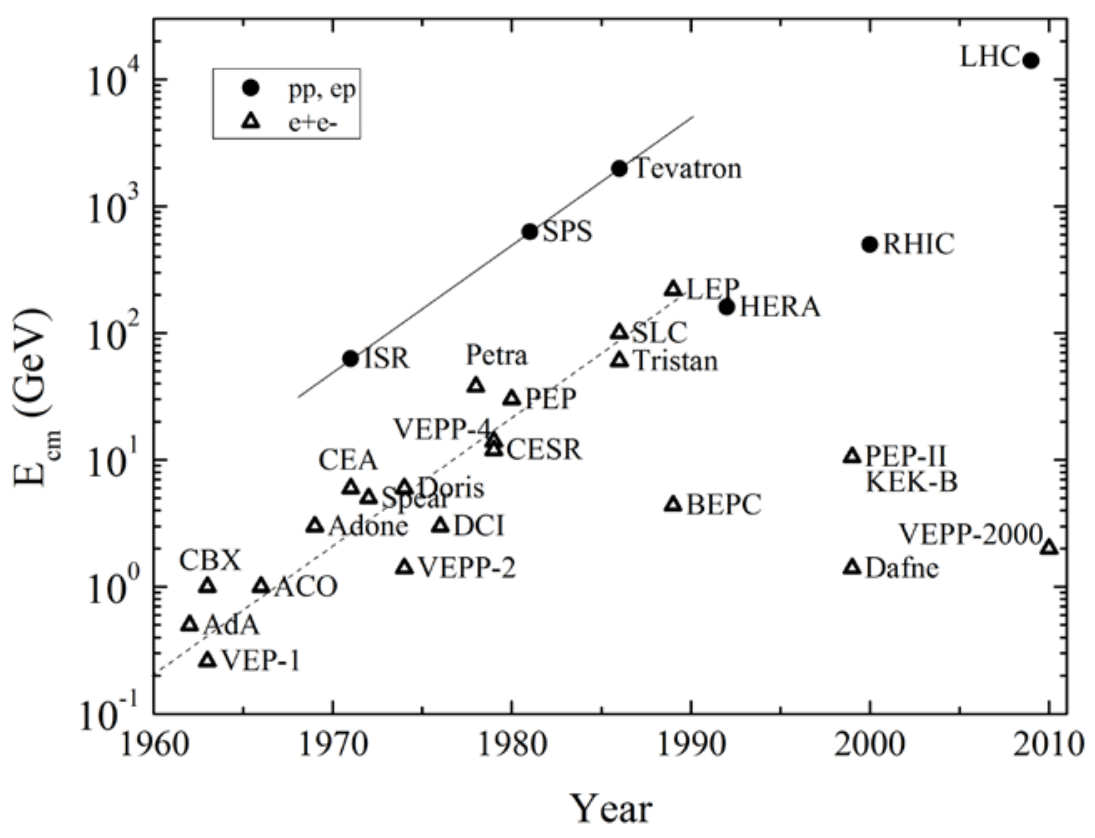

Figure 1: Progress of the energy of particle colliders over the decades.

\section{COLLIDERS BEYOND 2030: POSSIBILITIES}

To get to the energies of interest within given footprint of $10 \mathrm{~km}$, one needs fast acceleration. At present, three main opportunities for high energy colliders are being actively discussed which can be schematically classified by the type of media used for acceleration - solid state structures, plasma and crystals. (Below we reference the most recent reviews.)

Acceleration in dielectric structures: Most of present day accelerators employ radiofrequency fields $\left(f_{\mathrm{RF}}<10-30\right.$ $\mathrm{GHz}$ ) in resonant normal-conducting or SC structures powered by conventional RF sources and are in general limited to gradients of $\sim 100 \mathrm{MV} / \mathrm{m}$ due to surface breakdown phenomena. Combination of direct beam excitation (or wakefields radiated by a short intense "driving" bunch of electrons propagating in a high impedance environment) and hard dielectric materials (quartz, diamond, garnets, etc - which are characterized by lower power losses and higher breakdown gradients than metals) for structure fabrication, allow accelerating gradients of $\sim 100$ $\mathrm{MV} / \mathrm{m}$ and $\sim 1 \mathrm{GV} / \mathrm{m}$ in microwave $O(10) \mathrm{GHz}$ and $\mathrm{THz}$ dielectric structures [3], correspondingly. Conceptually, a dielectric wakefield accelerator (DWA)-based linear collider would consist of a large number of $\sim 100 \mathrm{GeV}$ modules (stages) with some $\sim 0.3 \mathrm{GeV} / \mathrm{m}$ gradient each driven by a separate $\sim 1 \mathrm{GeV}$ high intensity electron beam. Even without going into difficulties associated with staging, cost and power considerations, it is hard to imagine that more than $3 \mathrm{TeV}$ c.m. energy DWA facility can fit within a $10 \mathrm{~km}$ site.

Further increase of the gradient to $\sim 1-3 \mathrm{GV} / \mathrm{m}$ is thought to be possible in $\mu \mathrm{m}$ scale dielectric structures driven by lasers operating in optical or near-infrared regime [4]. In various options either external fiber lasers are coupled to the structures or semiconductor lasers can be integrated on the same slab right next to the microcells they 
power. Advantage of such an approach is that laser power sources can operate at very high repetition rates of $~ 10$ $100 \mathrm{MHz}$, that helps to get higher luminosity but again, a $10 \mathrm{TeV}$ c.m. energy collider will require $\sim 12 \mathrm{~km}$ of the total linac length [5]. To be of note that staging of sequential accelerating modules made of smaller size structures (microns vs millimeters) will require proportionally tighter synchronization, alignment and mechanical stability tolerances to keep beam trajectories and emittances under control.

Acceleration in plasma: In the past decade plasma-wakefield acceleration (PWA) methods has become of great interest because of the promise to sustain extremely large acceleration gradients. Electric fields due to charge separation in dense plasma are of the order of

$$
E_{0}=\frac{m_{e} c \omega_{p}}{e} \approx 100\left[\frac{G e V}{m}\right] \cdot \sqrt{n_{0}\left[10^{18} \mathrm{~cm}^{-3}\right]},
$$

where $\omega_{p}=\left(4 \pi n_{0} e^{2} / m_{e}\right)^{1 / 2}=2 \pi c / \lambda_{p}$ is the electron plasma frequency, $n_{0}$ is the ambient electron number density, $\lambda_{p} \approx 30[\mu \mathrm{m}] \cdot\left(n_{0}\left[10^{18} \mathrm{~cm}^{-3}\right]\right)^{1 / 2}$ is the plasma wavelength which sets the characteristic scale length of the wakefield. For example, generation of the PWA gradients on the order of $30-100 \mathrm{GV} / \mathrm{m}$ at plasma densities of $n_{0}=10^{17}-10^{18} \mathrm{~cm}^{-3}$ have been already demonstrated in small scale (few $\mathrm{cm}$ to a meter) experiments [5]. There are two ways to separate electrons and ions in the plasma - by lasers and by external beams. In the laser-plasma accelerators, a longitudinal accelerating electric field is generated by the ponderomotive force of an ultraintense laser pulse with duration on the order of the plasma period. This force, proportional to the gradient of the laser intensity, pushes the plasma electrons out of the laser beam path, separating them from the less mobile ions. This creates a travelling longitudinal electric field, in the wake of the laser pulse, with a phase velocity close to the speed of light - i.e., as needed for accelerating relativistic particles. The structure of the wakefield has broad phase space where negatively charged particles can be both accelerated and focused. Depletion of the laser energy determines the energy gain and the length of a single acceleration stage after which a new laser pulse must be coupled into plasma for further acceleration. Practical considerations indicate that this stage-to-stage distance is on the order of $1 \mathrm{~m}$, and minimization of the total linac length requires operation at relatively low densities $n_{0} \sim 10^{17} \mathrm{~cm}^{-3}$ and energy gain per stage of $10 \mathrm{GeV}$ and average gradient $\sim 5 \mathrm{TeV} / \mathrm{km}[6]$.

Alternative LWA concept involves the passage of an ultra relativistic electron bunch through a stationary plasma (either pre-formed by ionizing a gas by a laser or through field-ionization by the Coulomb field of the relativistic electron bunch itself). The plasma electrons are repelled by the bunch. Generating large amplitude wakefields requires short high density electron bunches compressed in all three spatial dimensions to sizes smaller than $\lambda_{p}-$ e.g., bunches of few $10^{10}$ electrons of few $\mu$ m's spot size and $\sim 10 \mu \mathrm{m}$ long. The wake produces a high gradient accelerating field Eq.(1) and transverse focusing for a negatively charged witness bunch behind the drive bunch [7]. The figure of merit in wakefield accelerators (plasma or dielectric) is the transformer ratio $R_{T}=$ (maximum accelerating field behind the drive bunch)/ (the maximum decelerating field inside the drive bunch) which is limited to $\sim 2$ for a finite length, longitudinally symmetric drive bunches and can reach somewhat reach higher values only for specially prepared triangular bunch current shapes. A proposed beam-PWA collider design [8] consists of a conventional $25 \mathrm{GeV}$ electron drive beam accelerator, which produces drive bunches distributed in counterpropagating directions to many (dozens to hundreds, depending on the final energy) one meter long plasma cells for both the electron and positron arms of the collider. Though each cell provides $25 \mathrm{GeV}$ energy gain $\left(R_{T} \approx 1\right)$, an average geometric accelerating gradient reaches only $0.25 \mathrm{TeV} / \mathrm{km}$ after taking into account some 100 meter long cell-to-cell gaps needed to bring up a fresh drive beam to the next cell from a single source of the high intensity 25 $\mathrm{GeV}$ electron bunches. As in other schemes, serious issues associated with staging and transfers from one cell to another (alignment, synchronization, etc) are anticipated. The number of stages can be significantly reduced if higher energy drive beams are available, e.g., some $0.6 \mathrm{TeV}$ energy gain might be possible in a single $\sim 400 \mathrm{~m}$ plasma cell driven by $1 \mathrm{TeV}$ protons if such high energy proton bunches can be compressed longitudinally to under $1 \mathrm{~mm}$ bunch length and kept tightly focused transversely [9]. Without going into practical considerations and just projecting such gradients further one can think of an ultimate $\sim 10 \mathrm{TeV}$ collider within $10 \mathrm{~km}$ footprint.

Acceleration in crystal channels: The density of charge carriers (conduction electrons) in solids $n_{0} \sim 10^{22-23} \mathrm{~cm}^{-3}$ is significantly higher than what was considered above in plasma, and correspondingly, the longitudinal fields of upto $100 \mathrm{GeV} / \mathrm{cm}$ or $10 \mathrm{TV} / \mathrm{m}$ are possible - see Eq.(1). The new effects at higher densities are due to intense energy radiation in high fields and increased scattering rates which result in fast pitch-angle diffusion over distances of $l_{d}$ $\sim 1[\mathrm{~m}] \cdot E[\mathrm{TeV}]$. The latter leads to particles escaping from the driving field; thus, it was suggested that particles are accelerated in solids along major crystallographic directions, which provide a channeling effect in combination with low emittance determined by an Angström-scale aperture of the atomic "tubes." Channeling with nanotubes is also 
being discussed. Positively charged particles are channeled more robustly, as they are repelled from ions and thus experience weaker scattering. Radiation emission due to betatron oscillations between the atomic planes is thought to be the major source of energy dissipation, and the maximum beam energies are limited to about $0.3 \mathrm{TeV}$ for positrons, $10^{4} \mathrm{TeV}$ for muons and $10^{6} \mathrm{TeV}$ for protons [10]. X-ray lasers can efficiently excite solid plasma and accelerate particles inside a crystal channel waveguide, though ultimate acceleration gradients $\sim 10 \mathrm{TeV} / \mathrm{m}$ might require relativistic intensities, exceeding those conceivable for x-rays as of today [11]. Moreover, only disposable crystal accelerators, e.g., in the form of fibers or films, are possible at such high externally excited fields which would exceed the ionization thresholds and destroy the periodic atomic structure of the crystal (so acceleration will take place only in a short time before full dissociation of the lattice). For the laser and plasma fields of about 1 $\mathrm{GV} / \mathrm{cm}=0.1 \mathrm{TV} / \mathrm{m}$ or less, reusable crystal accelerators can probably be built which can survive multiple pulses [12]. Side injection of powerful x-ray pulses into continuous fiber of $0.1-10 \mathrm{~km}$ long fiber allows to avoid multiple staging issues intrinsic to other methods and reach 10-1000 TeV energies, if the imperfections like crystal dislocations are kept under control and unintended crystal curvatures are less than inverse "critical" radius $R_{c}$ $\sim 2[\mathrm{~m}] \cdot E[\mathrm{TeV}]$ - so the channeling conditions remain [13].

It is to be noted that due to the imposed facility footprint limit, limited bending fields available and troubles with synchrotron radiation losses, the circular colliders do not seem conceivable for ultra-high c.m. energies $\sim 10$ 100 times the LHC and one with necessity comes to a linear configuration. Out of the purely energy gain arguments, heavier particles are preferred in all novel acceleration methods because they radiate less and one might argue that acceleration of electrons and positrons beyond $\sim 1-3 \mathrm{TeV}$ is impractical. Also, even at that energy, the c.m. energy spread due to beamstrahlung of electrons at the interaction point becomes prohibitively large at any other practical beam parameters and special measures will need to be taken, e.g., conversion of electrons and positrons in high energy $\gamma$-quanta and $\gamma-\gamma$ collisions [14].

Table 1: Far-reach particle colliders

\begin{tabular}{|l|l|l|l|}
\hline & Dielectric based & Plasma based & $\begin{array}{l}\text { Crystal } \\
\text { channeling }\end{array}$ \\
\hline Accelerating media & microstructures & ionized plasma & solid crystals \\
\hline $\begin{array}{c}\text { Energy source: option 1 } \\
\text { option 2 }\end{array}$ & $\begin{array}{l}\text { optical laser } \\
e^{-} \text {bunch }\end{array}$ & $\begin{array}{l}e^{-} \text {bunch } \\
\text { optical laser }\end{array}$ & x-ray laser \\
\hline Preferred particles & any stable & $e^{-}, \mu$ & $\mu^{+}, p^{+}$ \\
\hline Max accelerating gradient & $1-3 \mathrm{GV} / \mathrm{m}$ & $30-100 \mathrm{GV} / \mathrm{m}$ & $0.1-10 \mathrm{TV} / \mathrm{m}$ \\
\hline c.m. energy reach in 10 km & $3-10 \mathrm{TeV}$ & $3-50 \mathrm{TeV}$ & $10^{3}-10^{5} \mathrm{TeV}$ \\
\hline \# stages/10 km: option 1 \\
option 2 & $10^{5}-10^{6}$ & $\begin{array}{l}\sim 100 \\
10^{3}-10^{4}\end{array}$ & $\sim 1$ \\
\hline
\end{tabular}

Out of the remaining options of heavier particles, protons seems to be the only choice for c.m. energies beyond $10^{4}$ $\mathrm{TeV}=10 \mathrm{PeV}$; while in the context of the next 2-3 decades, when we do not envision energies of practical realization greater than $1 \mathrm{PeV}$, muons are much more attractive option because i) they are point-like particles und, contrary to protons, do not carry an intrinsic energy spread of elementary constituents; and ii) they do not have issues associated with nuclear interactions with accelerating media of plasma or solids. The fact that muons are not stable particles and decay as $d N / d t=-N / \gamma \tau_{0}$ becomes irrelevant in the fast acceleration schemes as the survived beam fraction at the final energy $E$ :

$$
\frac{N}{N_{0}} \approx\left(\frac{m_{\mu} c^{2}}{E}\right)^{\kappa},
$$

is very close to 1 as soon as the exponent $\kappa=\left(m_{\mu} c / \tau_{0} G\right)<<1 / \ln \left(E / m_{\mu} c^{2}\right)$ or, conversely, the average accelerating gradient $G>>3 \mathrm{MeV} / \mathrm{m}$ - the condition that easily holds for any scheme considered above (see Table 1 ). 


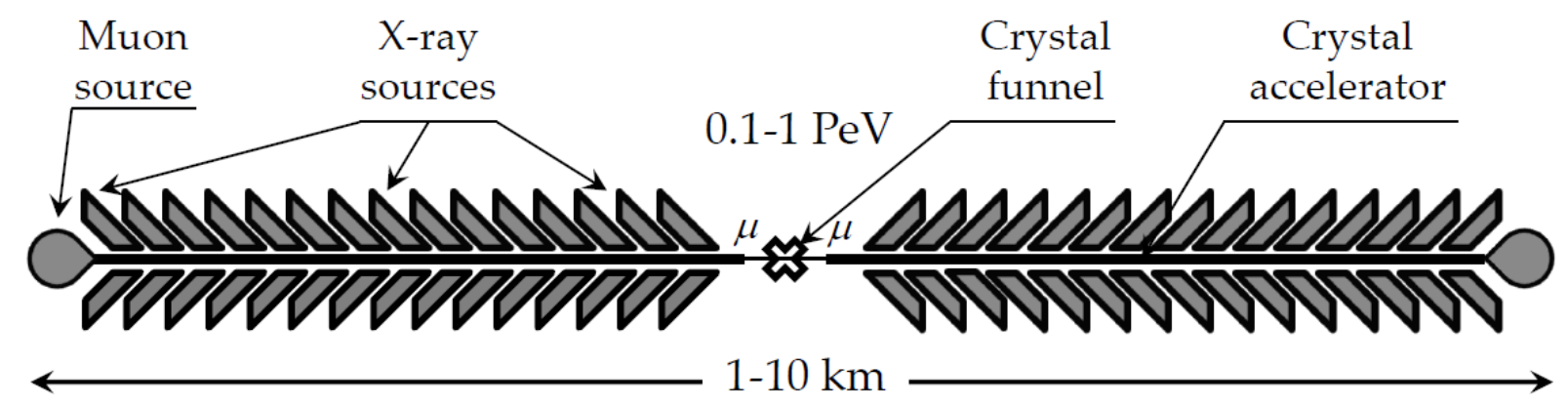

Figure 2: Concept of a linear X-ray crystal muon collider.

Following Ref.[52], one can explore possible luminosity reach of the ultimate high energy collider with $E_{\mathrm{cm}}>100 \mathrm{TeV}$ (see Fig.2). Given that acceleration of heavy particles in solid media/crystals is the technology of choice, it seems to be reasonable to limit the minimal overlap area of the colliding beams to the crystal lattice cell size $A \sim 1 \AA^{2}=10^{-16} \mathrm{~cm}^{-2}$ and to assume that the crystals of each collider arm will be aligned channel to channel. The other factor in the luminosity formula is the number of particles $N$ which can not be made arbitrary high due to the beam loading effect. Effective acceleration with transfer ratio $R_{T} \sim 1$ is possible only if the number of particles in the beam does not exceed the number of particles in the plasma volume excited by external source. Such volume is about $100 \lambda_{p}$ (longitudinal extent before excitation decays) $\times \lambda_{p}^{2}$ that results in $N_{0} \sim 10^{3}$ particles per individual bunch. Of course, exciting many parallel atomic channels $n_{c h}$ will proportionally increase the luminosity $L=f \cdot N^{2} / A=f$ $\cdot 10^{16} \cdot 10^{6} \cdot n_{c h}\left[\mathrm{~cm}^{-2} \mathrm{~s}^{-1}\right]$ - which can reach $10^{30} \mathrm{~cm}^{-2} \mathrm{~s}^{-1}$ at, e.g., $f=10^{6} \mathrm{~Hz}$ and $n_{c h} \sim 100$. Exceeding the value of the product $f n_{c h}$ beyond $10^{8} \mathrm{~Hz}$ can be very costly as the total beam power $P=f n_{c h} \cdot N E$ will exceed 16 MW per beam which we consider a practical limit from our collider cost considerations (see above). It might be beneficial instead to attempt to combine (focus) all the channeling beams into one using some kind of crystal funnel and, thus, gain a factor of $n_{c h}$ in the luminosity. Overall, in the power-limited scenario, the luminosity scales at very high energies as:

$$
L\left[\mathrm{~cm}^{-2} \mathrm{~s}^{-1}\right] \approx 4 \cdot 10^{33 \div 35} \times \frac{P^{2}[\mathrm{MW}]}{E^{2}[\mathrm{TeV}] \cdot f n_{c h}\left[10^{8} \mathrm{~Hz}\right]}
$$

The performance of the ultimate energy colliders can not increase with energy - either it is independent of it (if total beam power is small) or falls as $\sim 1 / E^{2}$. This fact, if not overturned by some future invention, indicates the need of the paradigm shift in the high energy particle physics because so far the performance goals of new energy frontier

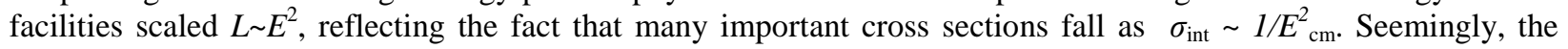
physics reach of colliders of the future will be limited to either resonances (phenomena with unusually high production rates at certain energies, indicating new particles) or other high cross-section reactions. Still, even the scaling as in Eq.(3) is much better than what other alternative sources of ultra-high energy particles can offer. For example, the number of cosmic ray events falls with the c.m. energy $E_{c m} \approx\left(2 E m c^{2}\right)^{1 / 2}$ even faster, approximately as $\sim 1 / E_{c m}^{7}$ and at the highest energies of $E_{c m} \sim 500 \mathrm{TeV}$, or $E \sim 10^{8} \mathrm{TeV}$, the event rates are extremely low - of about few per week even for the largest area observatories.

\section{DISCUSSION AND CONCLUSIONS}

The colliding beam method was a smashing success so far - almost 3 dozens colliders have been built over the past half-century and c.m. energies of about $10 \mathrm{TeV}$ have been achieved. At the same time, the pace of the energy progress has greatly slowed down due to increasing size, complexity and cost of the facilities, and as the result, the number of colliders currently in operation is about half of what we had 20 years ago. The prospects of facilities for the next 20 years are not very clear today, they will be dependent on the discoveries at the current machines, foremost, at the LHC.

It seems that economic realities will impose severe constrains for any far-future collider beyond 2030 to be built under about 10B\$ at current price, within footprint of some $10 \mathrm{~km}$, and with total electric power consumption 
of 10's to 100MW. As discussed above, there are possibilities which even currently conceivable methods can offer to reach ultra high energies on the order of $100-1000 \mathrm{TeV}$ within the abovementioned limits. The quest for the energy will come at the price of the expected luminosities and will require at least three paradigm shifts : 1 ) development of the new technology based on ultrahigh acceleration gradients $\sim 0.1-10 \mathrm{TeV} / \mathrm{m}$ in crystals; 2) acceleration of heavier particles, preferably, muons; and 3) new approaches to physics research with luminosity limited to $\sim 10^{30-32} \mathrm{~cm}^{-2} \mathrm{~s}^{-1}$.

As any other shift in the mainstream accelerator technology, the required switch to acceleration of muons in linear crystal structures will take a decade or two for an R\&D program to address several key issues: a) development of economical high-intensity coherent $\mathrm{X}$-ray sources, e.g., based on table-top $\sim \mathrm{GeV}$ scale electron accelerators [15, 16]; b) understanding the most effective mechanisms of coupling the X-ray power to the excitation of the lattice that can probably be studied even at the existing high power coherent X-ray sources, like LCLS in the US or Spring-8 in Japan; c) efficient production, injection and manipulation of nm-size muon beams (this program can effectively gain momentum out of the current research toward a muon collider); d) methods of combination of multiple crystal channeling beams into one (experiments at existing high energy proton machines might provide an important input there). A unique opportunity to explore most of these issue offers ASTA (Advanced Superconducting Test Facility), which is currently being built at Fermilab. It will be based on 0.7-1.5 GeV SC RF linac producing high brightness and high peak and high average power electron beams. The electrons can be used both for X-ray generation and muon production - the key components needed for the R\&D toward muon crystal accelearors. As in the past, as soon as the main technology issues are address, a great boost to the new development can be given by a test facility where the new acceleration methods are used for exploration of interesting "lowenergy" physics - e.g., a "table-top" factory of $\omega, \psi, \tau$ or even $Z, W$ or Higgs particles with decent luminosity and relatively low cost.

\section{ACKNOWLEDGMENTS}

The author wishes to thank C.Hill, A.Valishev, E.Eichten and S.Henderson for helpful discussions. Fermilab is operated by Fermi Research Alliance, LLC under Contract No. DE-AC02-07CH11359 with the United States Department of Energy.

\section{REFERENCES}

1. 2012 Global R\&D Funding Forecast Batelle R\&D Magazine (Dec. 2011)

2. V.Shiltsev, arxiv:1205.3087

3. W.Gai, in AIP Conf. Proc. 10863 (2008)

4. P.Bermel, et al., ICFA Beam Dynamics Newsletter 5691 (2011)

5. W.Leemans, ICFA Beam Dynamics Newsletter 5610 (2011)

6. C.Schroeder, et al., Phys. Rev. ST Accel. Beams 131013014 (2010)

7. P.Muggli, in AIP Conf. Proc. 129952 (2010)

8. A.Seryi, Nucl. Instr. Meth A 62323 (2010)

9. A.Caldwell, K.Lotov, A.Pukhov, F.Simon, Nature Phys. 5363 (2009)

10. I.Dodin, N.Fisch, Phys. Plasmas 15103105 (2008)

11. T.Tajima, M.Cavenago, Phys. Rev. Lett. 591440 (1987)

12. P.Chen, R.Noble, AIP Conf. Proc. 398273 (1997)

13. V.Biryukov, Yu.Chesnokov, V.Kotov, Crystal Channeling and Its Application at High Energy Accelerators (SpringerVerlag, Berlin, Heidelburg, 1997)

14. V.Telnov, arxiv:0908.3136

15. H.Schlenvoigt, et al., Nature Phys. 4130 (2008)

16. S.Kneip, et al., Nature Phys. 6980 (2010)

17. Program of Advanced Accelerator R\&D Users' Facility and Research Opportunities at Fermilab's Advanced Superconducting Test Accelerator (ASTA), unpublished, Fermilab (2012) 